\title{
Quantitative Analysis of Step-Growth Polymers by Size Exclu- sion Chromatography
}

\author{
Josef Brandt, ${ }^{\dagger}$ Naomi L. Haworth,${ }^{\S}$ Friedrich Georg Schmidt, ${ }^{\perp}$ Brigitte Voit ${ }^{\dagger}$, Michelle L. Coote, ${ }^{* \S}$ \\ Christopher Barner-Kowollik, $* \$$ Albena Lederer*†
}

‘Leibniz-Institut für Polymerforschung Dresden e.V., Hohe Straße 6, 01069 Dresden and Technische Universität Dresden, 01062 Dresden, Organische Chemie der Polymere, Germany

${ }^{\S}$ ARC Centre of Excellence for Electromaterials Science, Research School of Chemistry, Australian National University, Canberra, ACT 2601, Australia

*Preparative Macromolecular Chemistry, Institut für Technische Chemie und Polymerchemie, Karlsruhe Institute of Technology (KIT), Engesserstr. 18, 76131 Karlsruhe, and Institut für Biologische Grenzflächen, Karlsruhe Institute of Technology (KIT), Hermann-von-Helmholtz-Platz 1, 76344 Eggenstein-Leopoldshafen, Germany

${ }^{\perp}$ Evonik Industries AG, Paul-Baumann-Straße 1, 45764 Marl, Germany

\begin{abstract}
KEYWORDS Temperature Dependent Size Exclusion Chromatography (TD-SEC), Diels-Alder reaction, molar mass distribution, step-growth polymers
\end{abstract}

\begin{abstract}
We report an advanced analysis protocol that allows to quantitatively study the course of step-growth reactions by size exclusion chromatography on the example of the depolymerization of a Diels-Alder polymer based on a furane/maleimide couple at elevated temperatures. Frequently occurring issues of molar mass calibrations and overlap of monomer with solvent signals are addressed for determining reliable molar masses. Thereby, even kinetic parameters (e.g., rate coefficients) can be derived that otherwise would require performing additional spectroscopic experiments. Our results confirm first order behavior of the rDA reaction with an activation energy of $33 \mathrm{~kJ} \mathrm{~mol}^{-1}$.
\end{abstract}

Step-growth polymers (SGPs) represent an important class of macromolecules: polyurethanes, polyamides, polyesters and polycarbonates have found their permanent place in our everyday life. ${ }^{1-4}$ Their properties are different to chain-growth polymers, due to a particularity in their polymerization mechanism: a step-growth reaction requires very high monomer conversions in order to yield polymers of a high degree of polymerization $\left(D P_{n}\right)$, according Equation 1:

$$
D P_{n}=\frac{1}{1-x} \quad \text { Equation } 1
$$

At low conversions, $x$ dimers and higher oligomers are predominantly formed. Simultaneously, the molar mass dispersity $\left(D_{m}\right)$ of the formed polymers increases linearly from 1.0 to $2.0 .{ }^{5}$ Hence, SGPs are usually more broadly distributed than chain-growth polymers. Carothers and Flory described these considerations on the basis of simple statistical models, assuming that only linear polymers are formed. ${ }^{5-7}$

The final properties of a step-growth polymer are highly dependent on its molar mass, the corresponding molar mass distribution (MMD), intramolecular cyclization ${ }^{8-10}$ and the topology $y^{8,9,11}$. Due to the strong increase of the molar mass at high conversions, it is important to control the polymerization processes carefully. Thus, detailed studies on the kinetics of the reactions are crucial. One often employed concept for elucidating the reactions kinetics is to determine the conversion of functional groups spectroscopically. ${ }^{12-14}$ Although techniques such as NMR, UV/Vis or IR spectroscopy allow fast investigations they require that the reacting groups feature characteristic absorptions and that robust absorption-concentration correlations can be assumed. Yet, even when all requirements are fulfilled, spectroscopic techniques only yield average values and do not reveal information about the MMD that can give further insights into the reaction mechanisms.
Size exclusion chromatography (SEC) is often used for deducing the MMD of polymers and calculating molar mass averages..$^{15-17}$ In principle, the conversion of functional groups can be calculated from the number average molar mass $\left(M_{n}\right)$ by calculating the $D P_{n}$ and applying Equation 1. This procedure, however, requires that molar masses can be determined very accurately, which is a particular challenge in the case of relatively small step-growth polymers: The separation mechanisms as well as the detector responses can become difficult to assess in the case of oligomers. ${ }^{18,19}$ As a consequence, particular care has to be taken during calibration and calculation of molar masses.

Herein we describe a SEC evaluation procedure that can be applied for reliable and comprehensive analyses of SGPs. As an example, a DielsAlder (DA) based step-growth polymer is investigated. At elevated temperatures the forward DA reaction experiences competition by the retro DA (rDA) process, leading to a depolymerization of the DA polymers. By using temperature dependent SEC (TD SEC), the depolymerization reaction can be monitored in situ. ${ }^{20,21}$ The optimum way to investigate a step-growth reaction in its forward or backward direction depends on the scientific objective to be studied. In many cases it might be more interesting to study the actual polymerization. The herein described procedure is valid for such studies, too, as long as regular stepgrowth statistics are not violated (or an alternative theoretical model for predicting the MMD can be constructed). In our case the characteristics of the $\mathrm{rDA}$ reaction are of interest. Under the assumption of a thermodynamic equilibrium between polymerization and depolymerization at each temperature the relation shown in Equation 1 holds for the $\mathrm{rDA}$ reaction, too.

In Scheme 1 the rDA reaction sequence of the investigated DA furanmaleimide system is depicted. Details concerning the synthesis of the DA polymer can be found in chapter 1 of the SI. 


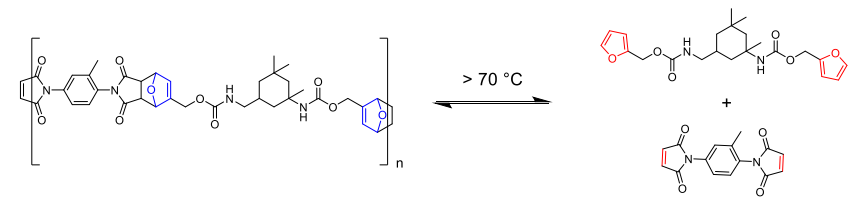

Scheme 1. rDA reaction scheme of the DA furan-maleimide under investigation.

A typical chromatogram of the DA furan-maleimide polymer is shown in Figure 1. A sample of the DA polymer was kept at $80^{\circ} \mathrm{C}$ in the autosampler of the TD SEC instrument and samples were drawn after different heating times (for more details refer to chapter 2 of the SI). Clearly, the entire molar mass distribution shifts towards higher elution volumes (i.e., lower molar masses) with increasing heating time, while discrete oligomer peaks can be distinguished. Thus, the qualitative proof of a depolymerization is given.

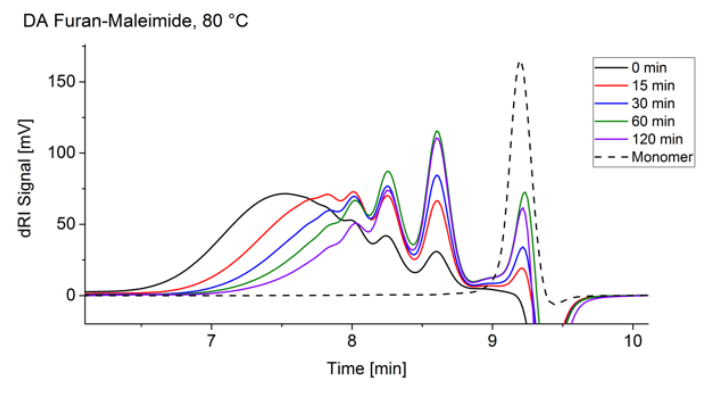

Figure 1. SEC chromatograms of the DA furan-maleimide system after different $\mathrm{rDA}$ times prior to injection at $80^{\circ} \mathrm{C}$ (acquired at 1 $\mathrm{mL} / \mathrm{min}$ ). The dashed trace indicates the chromatogram of the individual furan-monomer.

For the evaluation of the chromatograms, a reliable molar mass determination is necessary. Absolute molar mass determination by static light scattering (SLS) is virtually impossible for molar masses in the oligomer range. Hence, a relative molar mass calibration has to be constructed. However, the use of narrowly distributed standards is problematic: Employing standards of a different type of polymer (e.g., PS or PMMA) leads to imprecise results and a universal calibration is difficult as the viscosity signal is also becoming very weak for small oligomers. Another issue is that the dRI signal (or an UV-absorption signal) is not necessarily proportional to concentration at low molar masses ${ }^{22}$, making the evaluation of viscosity signals (the intrinsic viscosity is the quotient of viscosity and concentration, $\left.[\eta]_{0} \sim \eta / c\right)^{19}$ and the calculation of molar mass averages inaccurate.

A solution to these problems can be found in the chromatograms themselves. In fact, the position of the oligomer peaks can be used for establishing a sample-specific calibration. During the course of a stepgrowth polymerization or depolymerization the oligomer peaks emerge. Their exact molar mass can be calculated from the molar mass of the monomer(s) and their position is readily determined. For a more reliable determination of the peak maxima, the dRI trace can simply be derived: In the second derivative $\left(\mathrm{d}^{2} d R I / \mathrm{d} t^{2}\right)$ each peak maximum is represented by a much sharper minimum (see Figure $2 \mathrm{~A}$ ). If the SEC setup features a sufficiently high plate number it should be possible to detect a variety of peaks that can be used for establishing a robust column calibration. In the case of separation according to size (absence of enthalpic interactions), a linear relation between $\log M$ and elution time should be found and the calibration can be extrapolated to higher molar masses (Figure 2B). Extrapolating a calibration curve has to be treated with care as unexpected deviations from the predicted behavior cannot be taken into account. Again, the low molar mass end is critical as deviations in separation behavior can occur due to end group effects. ${ }^{18}$ If, however, good linearity of the calibration is found close to the low molar mass limit and the column generally allows measuring significantly larger polymers (the used ResiPore column allows separating polymers up to $1 \mathrm{MDa}^{20}$ ) it is very likely that the molar mass calibration continues in a linear manner. It is generally possible to establish good SEC conditions for the separation for most polymers by choosing appropriate solvent-column combinations (i.e., minimization of enthalpic interactions between stationary phase and analyte).
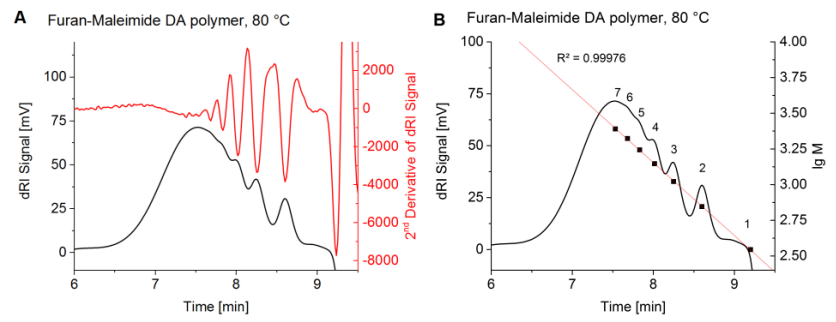

Figure 2. A: SEC chromatogram of the DA polymer at $80^{\circ} \mathrm{C}$ in DMSO (acquired at $1 \mathrm{~mL} / \mathrm{min}$ ) and the 2 nd derivative of the $\mathrm{dRI}$ signal. B: Resulting calibration curve, indicating good separation according to size. (The numbers indicate the $D P_{\mathrm{n}}$ of the respective peaks; the position of the monomer peak is taken from an individual measurement of the monomer).

In a conventional data analysis procedure, the calibration is used for calculating the molar masses of each slice in the chromatogram and determining the molar mass averages. Two aspects make this procedure prone to errors. Firstly, due to its low molar mass it can easily occur that the peak of the monomer is partially or completely occluded by the solvent peak. As it can be seen from Figure 1 and Figure 2A, peak overlap was the case in our analyses. Consequently, it is not possible to include the monomer peak in the calculation of any molar mass average. When the monomer peak does not contribute to the molar mass average the obtained results are biased towards high values. Especially at high rDA conversions, when a substantial fraction of monomers is present, the error is becoming significant. Secondly, the non-constant $\mathrm{d} n / \mathrm{d} c$ at very low molar masses leads to incorrect molar mass averages as the molar ratios of the oligomers are not correctly represented by their peak area. ${ }^{18,19}$

A more elegant evaluation protocol is to predict the MMD of the stepgrowth polymer as a function of the monomer conversion. The MMD can be predicted for any arbitrary monomer conversion according to Equation 2. The number fraction for an arbitrary $D P_{n}\left(n\left(D P_{n}\right)\right)$ can be calculated from the probability to find a polymer bond $p$ ( $p$ equals the monomer conversion $x)$. Finally $n\left(D P_{n}\right)$ can be converted into the corresponding weight fraction $w\left(D P_{n}\right)$ by multiplication with the mass of the respective $D P_{n}$. By varying $p$ the theoretical MMD can be fitted to the experimentally acquired chromatogram and, thus, the monomer conversion can be obtained.

$$
n_{D P_{n}}=p^{\left(D P_{n}-1\right)} \cdot(1-p) \quad \text { Equation 2 }
$$

The theoretical MMD can subsequently be fitted to the experimentally acquired MMD in order to derive its monomer conversion. In Figure 3 the result of this procedure is shown on the example of the $\mathrm{rDA}$ reaction of the furan-maleimide couple at $80{ }^{\circ} \mathrm{C}$. A detailed description of the fitting procedure can be found in chapter 3 of the SI.

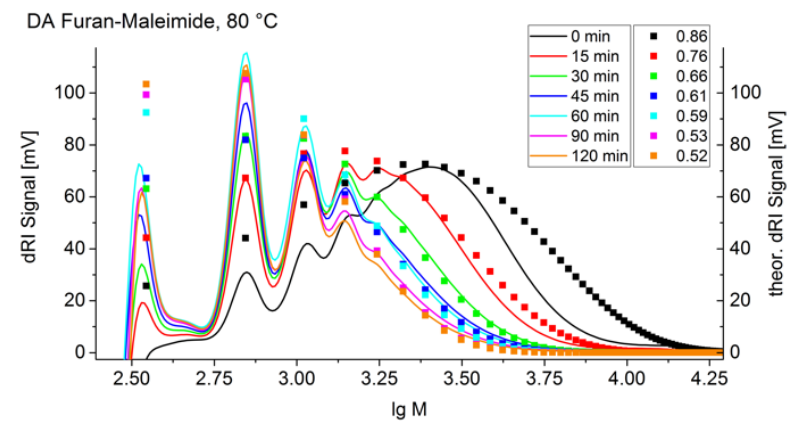

Figure 3. Acquired MMDs of the DA polymers at $80{ }^{\circ} \mathrm{C}$. The left axis represents the dRI signal after different $\mathrm{rDA}$ reaction times 
(solid lines), whereas the right axis gives the theoretically determined MMD at the indicated conversions (symbols).

It is evident that the predicted MMDs describe the experimentally obtained MMDs very well in most of the cases. Especially at high conversions (i.e., large $p$ ), however, the experimentally acquired MMDs are significantly narrower than the predicted ones. These discrepancies indicate either invalid assumptions in the statistical description of the step-growth reaction or deviations of the calibration curve at high molar masses. As already discussed above, the calibration curve can be considered reliable. On the other hand, Equation 2 is only valid if exclusively linear polymers are formed, if perfect stoichiometry of both monomers is guaranteed and if the formation (or scission) of bonds occurs randomly. Ring formation ${ }^{23-26}$, incomplete conversion due to nonstoichiometry or preferential bond scission in the middle of polymers ${ }^{27}$ could contribute to the deviations at high molar masses but more indepth studies would be required for reliable explanations. After longer rDA reaction times, experiment and theory fit better, although some deviations are still found in the low molar mass regime. These deviations are most likely due to the non-constant $\mathrm{d} n / \mathrm{d} c$ of the oligomers and the corresponding non-proportionality of dRI signal and concentration.

For assessing the validity of the procedure, the obtained conversions (i.e., bonding probabilities $p$ ) were transformed into \%Bonding Decrease according to Equation 3 ( $p_{0}=$ bonding probability before rDA reaction, $p=$ bonding probability after certain $\mathrm{rDA}$ time). The \%Bonding Decrease value ranges from 0 to $100 \%$ and represents the extent of the $\mathrm{rDA}$ reaction $(0 \%=$ no debonding, $100 \%=$ total debonding $)$. It can be alternatively calculated according to Equation 4, which was obtained by substituting Equation 1 into Equation 3 (conversion $x=$ bond probability $p$ ). The latter equation is more suitable for calculating the $\%$ Bonding Decrease following the conventional approach of calculating the $M_{n}$ of the sample and converting it into the $D P_{n}$ (index rDA = after $\mathrm{rDA}$ reaction, index $0=$ before $\mathrm{rDA}$ reaction). ${ }^{28}$

$$
\begin{array}{ll}
\% \text { Bonding Decrease }=\frac{p_{0}-p}{p_{0}} \cdot 100 \% & \text { Equation } 3 \\
\% \text { Bonding Decrease }= & \\
\left(1-\frac{1-\frac{1}{D P_{n, r D A}}}{1-\frac{1}{D P_{n, 0}}}\right) \cdot 100 \% & \text { Equation } 4
\end{array}
$$

In Figure 4A, the \%Bonding Decrease that was obtained from fitting the MMD and applying Equation 3 is plotted for different rDA temperatures and reaction times. Apparently, the obtained values represent the rDA progress in a reasonable manner, as more debonding is achieved at higher temperatures. For a better comparison, the highest achieved $\%$ Bonding Decrease values of each temperature are compiled in Figure $4 \mathrm{~B}$; together with the values for the conventional approach of calculating the $M_{n}$ and applying Equation 4. A third dataset is included that shows the theoretically expected \%Bonding Decrease of a furan-maleimide dimer, as obtained from $a b$ initio quantum mechanical calculations using procedures we have validated previously for Diels-Alder polymers (refer to SI for further information). ${ }^{28-32}$
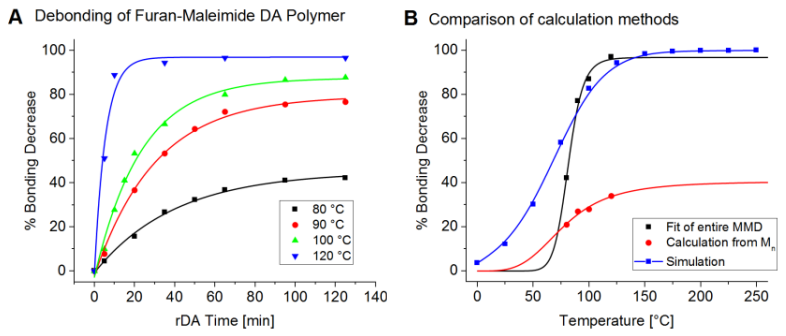

Figure 4. Time resolved \%Bonding Decrease of the DA polymers (A) and comparison of the equilibrium \%Bonding Decrease determined from fitting the entire MMD, calculating the $M_{n}$ and the simulation of a furan-maleimide dimer (B). The lines represent a guide for the eye of the expected temperature dependency of the bonding decrease.

Clearly, the values from calculating the $M_{n}$ appear substantially too low; they tend towards a final \%Bonding Decrease close to $40 \%$, although the chromatograms that are obtained at 100 and $120{ }^{\circ} \mathrm{C}$ indicate almost complete debonding (refer to Figure S4 and S5 in the SI). This is a direct consequence of the overestimation of the $M_{n}$ as the monomer peak could not be included into the calculation. Hence, the minimum $D P_{n}$ that can be obtained is 2 . Applying Equation 4 with $D P_{n, 0}=20$ and $D P_{n, r D A}=2$ results in $\%$ Bonding Decrease $=47 \%$, thus explaining the systematically too low values in Figure 4B. The \%Bonding Decrease values from fitting the MMD are significantly higher and correspond much better to the qualitative impression of the rDA progress (Figure $\mathrm{S} 4$, Figure S5). At temperatures lower than $70^{\circ} \mathrm{C}$, the data from the $M_{n}$ calculations still predict a significant portion of debonding, whereas the data from the MMD fit approaches $0 \%$ Bonding Decrease at these temperatures. Again, the latter case is more realistic, as the synthesis of the DA polymer was performed at $70{ }^{\circ} \mathrm{C}$. The values from fitting the MMD resemble the $\mathrm{S}$-shaped curve that is predicted from the furan-maleimide dimer simulation better than the values from calculating the $M_{n}$. The simulation predicts similar \%BondingDecreases, though at very low temperatures it over-estimates the extent of debonding.

The validity of the obtained values was additionally assessed by investigating the kinetics of the rDA reactions in more detail. In literature it is described that rDA reactions usually follow a first order kinetic behavior. ${ }^{13,33}$ Hence, if our values are true, first order behavior should be observed in our case, too. In Figure 5A, the conversions derived by fitting the chromatograms are plotted in a first order kinetic plot $(\ln ((p-$ $\left.\left.p_{e q}\right) /\left(p_{0}-p_{e q}\right)\right)$ over $r D A$ time, with $p_{e q}=$ equilibrium $p$ at the given temperature and $p_{0}=$ initial $p$ before any $\mathrm{rDA}$ reaction). In fact, the data points coincide well with a linear fit with the slope of the negative reaction rate coefficient. The dataset at $120^{\circ} \mathrm{C}$ suffers from strong scattering of the values as almost $100 \% \mathrm{rDA}$ conversion is achieved and consequently large errors result from small inaccuracies of the conversion data. Having determined the rate coefficients at variable temperatures enables deriving the reaction activation energy $E_{A}$ from an Arrhenius plot (Figure $5 \mathrm{~B}$ ). The obtained $E_{A}$ of $33 \mathrm{~kJ} / \mathrm{mol}$ is close to values reported in literature (e.g., $21 \mathrm{~kJ} / \mathrm{mol}$ for a crosslinking furan-maleimide system $)^{13,34,35}$ and, thus, appears reasonable. The precision of the determined rate coefficients is approx. $\pm 5 \%$ and of the activation energy approx. $\pm 3 \%$, respectively (see SI chapter 5 for details on the error estimation)
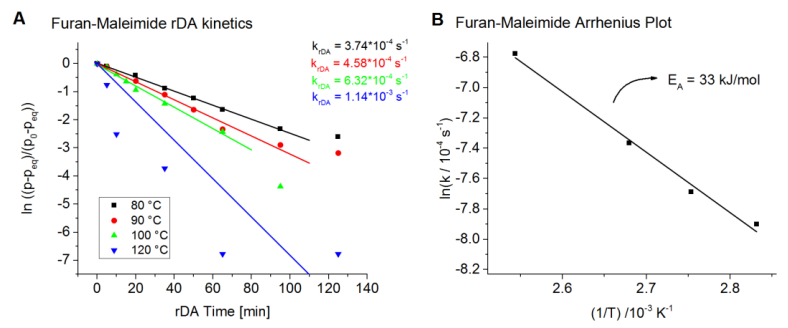

Figure 5. First order reaction plot of the furan-maleimide rDA reaction that allows determining the reaction rate coefficients (A) and the resulting Arrhenius plot that indicates the activation energy of the $\mathrm{rDA}$ reaction $(\mathrm{B})$

In conclusion, we introduce a novel approach for quantitatively evaluating the polymerization or depolymerization of SGPs by SEC. Typical problems of imprecise column calibrations, biased molar mass averages due to unreliable dRI signals in the oligomer region and the risk of overlapping solvent peaks are overcome by a simple self-calibration approach and fitting of the MMD according to step-growth statistics. It shall be noted that the herein described procedure is not only valid for investigating DA polymers by TD SEC. Indeed, similar issues are expected whenever SGPs containing significant amounts of lower oligomers are studied by any SEC method with simple dRI detection. Provided, oligomeric peaks are visible, this self-calibration procedure 
provides a simple general approach to treating these problems without the use of additional expensive analysis techniques.

Being able to monitor the progress of polymerization or depolymerization reactions quantitatively (in our example at elevated temperatures) allows deriving kinetic parameters that otherwise would require additional spectroscopic experiments. Hence, the potential of the standard SEC methodology is exploited further, as robust results can be obtained even from problematic samples such as relatively small step-growth oligomers and polymers.

\section{ASSOCIATED CONTENT}

Supporting Information. All details concerning the exact synthesis and evaluation procedures (including a fitting script), additional chromatograms and information about the quantum chemical calculations can be found in the supporting information. This material is available free of charge via the Internet at http://pubs.acs.org.

\section{AUTHOR INFORMATION}

\section{Corresponding Author}

*E-Mail: lederer@ipfdd.de

*E-Mail: christopher.barner-kowollik@kit.edu

*E-Mail: michelle.coote@anu.edu.au

\section{Author Contributions}

All authors have given approval to the final version of the manuscript.

\section{Funding Sources}

The study was supported by Evonik Industries AG.

\section{Notes}

The authors declare no competing financial interest.

\section{ACKNOWLEDGMENT}

The DA furan-maleimide polymer was kindly provided from $\mathrm{K}$. Öhlenschläger (Karlsruhe Institute of Technology). A. L., C.B.-K. and M. L. C. are thankful for funding from Evonik Industries AG. C.B.-K. acknowledges continued funding from the Karlsruhe Institute of Technology (KIT) in the context of the STN and BIFTM programs of the Helmholtz association. M. L. C. thanks the Australian National Computational Infrastructure for generous allocations of supercomputing time.

\section{ABBREVIATIONS}

DA, Diels-Alder; DMSO, dimethyl sulfoxide; $\mathrm{DP}_{\mathrm{n}}$, degree of polymerization; dRI, differential refractive index; IR, infrared; MMD, molar mass distribution; NMR, nuclear magnetic resonance; p, probability; PMMA, poly(methyl methacrylate); PS, polystyrene; rDA, retro Diels-Alder; SEC, size exclusion chromatography; SGP, step-growth polymer(ization); SI, supporting information; TD SEC, temperature dependent size exclusion chromatography; UV/Vis, ultraviolet/visible

\section{REFERENCES}

(1) Rogers, M. E.; Long, T. E.; Turner, S. R. Synthetic Methods in Step-Growth Polymers; Rogers, M. E., Long, T. E., Eds.; John Wiley \& Sons, Inc.: Hoboken, NJ, USA, 2003.

(2) Randall, D.; Lee, S. The Polyurethanes Book; John Wiley \& Sons, 2003.
(3) Deopura, B. L.; Alagirusamy, R.; Joshi, M.; Gupta, B. Polyesters and Polyamides; Woodhead Publishing Limited: Cambridge, 2008.

(4) LeGrand, D. G.; Bendler, J. T. Handbook of Polycarbonate Science and Technology; Marcel Dekker, Inc.: New York, 2000.

(5) Flory, P. J. J. Am. Chem. Soc. 1936, 58 (10), 1877-1885.

(6) Flory, P. J. J. Am. Chem. Soc. 1939, 61 (12), 3334-3340.

(7) Carothers, W. H. J. Am. Chem. Soc. 1929, 51 (8), 2548-2559.

(8) Kricheldorf, H. R. Acc. Chem. Res. 2009, 42 (8), 981-992.

(9) Chen, H.; Kong, J.; Tian, W.; Fan, X.-D. Macromolecules 2012, 45 (15), 6185-6195.

(10) Chen, H.; Kong, J. Polym. Chem. 2016, 7 (22), 3643-3663.

(11) Chen, H.; Zhang, S.; Kong, J. Polym. Chem. 2015, 6 (6), 909916.

(12) Goiti, E.; Heatley, F.; Huglin, M. B.; Rego, J. M. Eur. Polym. J. 2004, 40 (7), 1451-1460.

(13) Goiti, E.; Huglin, M. B.; Rego, J. M. Macromol. Rapid Commun. 2003, 24 (11), 692-696.

(14) Zhang, Y.; Broekhuis, A. A.; Picchioni, F. Macromolecules 2009, 42 (6), 1906-1912.

(15) Podzimek, S. Light Scattering, Size Exclusion Chromatography and Asymmetric Flow Field Flow Fractionation; WILEY: Hoboken, New Jersey, 2011.

(16) Striegel, A. M.; Yau, W. W.; Kirkland, J. J.; Bly, D. D. Modern Size-Exclusion Liquid Chromatography, 2nd ed.; John Wiley \& Sons: Hoboken, New Jersey, 2009.

(17) Kostanski, L. K.; Keller, D. M.; Hamielec, A. E. J. Biochem. Biophys. Methods 2004, 58 (2), 159-186.

(18) Striegel, A. M. Anal. Bioanal. Chem. 2013, 405 (28), 8959-8967.

(19) Chance, R. R.; Baniukiewicz, S. P.; Mintz, D.; Ver Strate, G.; Hadjichristidis, N. Int. J. Polym. Anal. Charact. 1995, I (1), 334.

(20) Brandt, J.; Guimard, N. K.; Barner-Kowollik, C.; Schmidt, F. G.; Lederer, A. Anal. Bioanal. Chem. 2013, 405 (28), 8981-8993.

(21) Brandt, J.; Oehlenschlaeger, K. K.; Schmidt, F. G.; BarnerKowollik, C.; Lederer, A. Adv. Mater. 2014, 26 (33), 5758-5785. Gridnev, A. A.; Ittel, S. D.; Fryd, M. J. Polym. Sci. Part A Polym. Chem. 1995, 33 (7), 1185-1188.

(23) Kricheldorf, H. R.; Schwarz, G. Macromol. Rapid Commun. 2003, 24 (56), 359-381.

(24) Kricheldorf, H. R. Macromol. Rapid Commun. 2009, 30 (16), 1371-1381.

(25) Proppe, J. Macromol. Theory Simul. 2015, 24 (5), 500-512.

(26) Kricheldorf, H. R. Macromol. Rapid Commun. 2008, 29 (21), 1695-1704.

(27) Pahnke, K.; Brandt, J.; Gryn'ova, G.; Lin, C. Y.; Altintas, O.; Schmidt, F. G.; Lederer, A.; Coote, M. L.; Barner-Kowollik, C. Angew. Chemie Int. Ed. 2016, 55 (4), 1514-1518.

(28) Guimard, N. K.; Ho, J.; Brandt, J.; Lin, C. Y.; Namazian, M.; Mueller, J. O.; Oehlenschlaeger, K. K.; Hilf, S.; Lederer, A.; Schmidt, F. G.; Coote, M. L.; Barner-Kowollik, C. Chem. Sci. 2013, 4 (7), 2752-2759.

(29) Pahnke, K.; Brandt, J.; Gryn'ova, G.; Lindner, P.; Schweins, R.; Schmidt, F. G.; Lederer, A.; Coote, M. L.; Barner-Kowollik, C. Chem. Sci. 2015, 6 (2), 1061-1074.

(30) Pahnke, K.; Haworth, N. L.; Brandt, J.; Paulmann, U.; Richter, C.; Schmidt, F. G.; Lederer, A.; Coote, M. L.; Barner-Kowollik, C. Polym. Chem. 2016, 7 (19), 3244-3250.

(31) Oehlenschlaeger, K. K.; Guimard, N. K.; Brandt, J.; Mueller, J. O.; Lin, C. Y.; Hilf, S.; Lederer, A.; Coote, M. L.; Schmidt, F. G.; Barner-Kowollik, C. Polym. Chem. 2013, 4 (16), 4348-4355. Zhou, J.; Guimard, N. K.; Inglis, A. J.; Namazian, M.; Lin, C. Y.; Coote, M. L.; Spyrou, E.; Hilf, S.; Schmidt, F. G.; BarnerKowollik, C. Polym. Chem. 2012, 3 (3), 628-639.

(34) Liu, Y. L.; Hsieh, C. Y.; Chen, Y. W. Polymer 2006, 47 (8), 2581-2586.

(35) Gandini, A.; Coelho, D.; Silvestre, A. J. D. Eur. Polym. J. 2008, 44 (12), 4029-4036. 